\title{
LINEAR PREDICTIVE RECEIVERS FOR PHASE-UNCERTAIN CHANNELS
}

\author{
Gianluigi Ferrari, Giulio Colavolpe, and Riccardo Raheli \\ Università degli Studi di Parma \\ Dipartimento di Ingegneria dell'Informazione \\ Parco Area delle Scienze 181A \\ 43100 Parma, Italy
}

\begin{abstract}
In this paper, we propose linear predictive receivers for phaseuncertain channels. These receivers are attractive from a conceptual viewpoint because they generalize previous solutions based on noncoherent sequence detection. On the practical side, the proposed algorithms lend themselves to the implementation of adaptive receivers capable of copying with possible time variations of the statistics of the underlying phase model.
\end{abstract}

\section{INTRODUCTION}

In future generation wireless local networks, the integration of mobile and satellite communications will call for detection algorithms suitable to phase-uncertain channels. In particular, these detection algorithms should be robust to oscillator instabilities, generating phase noise, and time-varying frequency offsets, possibly due to the Doppler shift experienced, for example, in low earth orbit (LEO) satellite systems [1].

In this paper, we derive a linear predictive receiver following the methods previously presented for fading channels [2-5]. The proposed algorithms compare favorably with previously appeared noncoherent sequence detection schemes [6], which exhibit high robustness to strong phase instabilities or uncompensated frequency offsets typically produced by the Doppler effect. We derive detection schemes based on a Viterbi algorithm (VA) for linear coded modulations. Results for soft-output forward-backward (FB) algorithms and for continuous phase modulations (CPM) are also described.

\section{LINEAR PREDICTIVE RECEIVERS}

We consider the lowpass complex equivalent system depicted in Fig. 1. We assume that a sequence of $K$ independent and uniformly distributed $M$-ary symbols $\left\{a_{k}\right\}_{k=0}^{K-1}$, denoted by the vector $a$ in the figure, feeds an encoder $/$ modulator, which can be modeled as a finite state machine with state $s_{k}$. We also define the relevant state transition as $t_{k} \triangleq$ $\left(a_{k}, s_{k}\right)$. The linearly modulated continuous-time signal

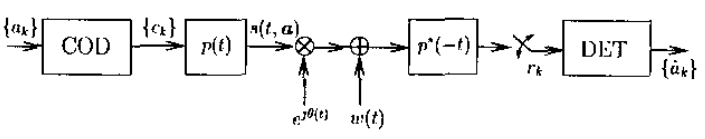

Figure 1: System model.

$s(t, \boldsymbol{a})$ is obtained by letting the code symbol $c_{k}$ be carried by a suitable shaping pulse $p(t)$. Although suboptimal in the presence of a time-varying channel, a matched-filter front-end with sampling rate of one sample per symbol can be practically used, provided the phase process is not affected by very strong variations [7]. The resulting observation model is

$$
r_{k}=c_{k} e^{j \theta_{k}}+n_{k}
$$

where $\left\{n_{k}\right\}$ is an independent identically distributed (i.i.d.) additive Gaussian noise sequence with variance $N_{0}$. The channel phase process $\theta_{k}$ is assumed stationary and zeromean, and the autocorrelation sequence of the phasor process $e^{j \theta_{k}}$ is denoted by $R_{\theta}(n) \triangleq E\left\{e^{j \theta_{k+n}} e^{-j \theta_{k}}\right\}$.

Should the phase process $\left\{\theta_{k}\right\}$ be known exactly, a coherent sequence detector based on the VA could be derived, with branch metric

$$
\gamma\left(t_{k}\right) \triangleq\left|r_{k}-e^{j \theta_{k}} c_{k}\right|^{2}=\left|c_{k}\right|^{2}\left|r_{k}^{\prime}-e^{j \theta_{k}}\right|^{2}
$$

where $r_{k}^{\prime} \triangleq r_{k} / c_{k}$ is a normalized observation. If only a statistical characterization of the phase process is available, a practical sequence detector can be obtained by using (2) with the exact value $\theta_{k}$ replaced by a suitable estimate $\widehat{\theta}_{k}$.

In the general case of a time-varying phase process, the data-aided minimum mean square error (MMSE) phase estimate based on $\nu$ previous observations is the conditional mean

$$
\widehat{\theta}_{k}=E\left\{\theta_{k} \mid \mathbf{c}_{k-\nu}^{k-1}, \mathbf{r}_{k-\nu}^{k-1}\right\}
$$

where an indexed vector notation is used to denote code symbols and observations from time $k-\nu$ to $k-1$. The expectation in (3) leads to a non-linear estimate, which is usually rather difficult to compute. 
Instead of directly estimating the phase, we use the following indirect estimation strategy. For large signal-to-noise ratio (SNR) $r_{k}^{\prime} \approx e^{j \theta_{k}}$, and then $\theta_{k} \approx \arg \left\{r_{k}^{\prime}\right\}$. In order to exploit the phase correlation characteristics in the estimation process, we consider a data-aided linear prediction $\widehat{r_{k}^{\prime}}$ of $r_{k}^{\prime}$ based on the previous $\nu$ normalized observations $\mathbf{r}_{k-\nu}^{\prime k-1}$, i.e.,

$$
\widehat{r_{k}^{\prime}}=\sum_{i=1}^{\nu} p_{i} r_{k-i}^{\prime}
$$

where $\left\{p_{i}\right\}_{i=1}^{\nu}$ are the prediction coefficients and $\nu$ is the prediction order.

The prediction coefficients in (4) can be computed by solving the MMSE problem [2-5]

$$
\min _{\left\{p_{i}\right\}_{i=1}^{\nu}} E\left\{\left|r_{k}^{\prime}-\sum_{i=1}^{\nu} p_{i} r_{k-i}^{\prime}\right|^{2} \mid \mathbf{c}_{k-\nu}^{k-1}\right\}
$$

which leads to a Yule-Walker linear system $\mathbf{R} \mathbf{p}=\mathbf{b}$, where $\mathbf{R}$ is a square $\nu \times \nu$ matrix whose elements have the following expression

$$
[\mathbf{R}]_{l, m}= \begin{cases}R_{\theta}(|l-m|) & \text { if } \quad l \neq m \\ R_{\theta}(0)+\frac{N_{0}}{\left|c_{b-l}\right|^{2}} & \text { if } l=m\end{cases}
$$

$\mathbf{p} \triangleq\left[p_{1} \cdots p_{\nu}\right]^{T}$ is the unknown vector and $\mathbf{b}=\left[R_{\theta}(1)\right.$, $\left.R_{\theta}(2), \cdots, R_{\theta}(\nu)\right]^{T}$.

The solution of this Yule-Walker system entitles us to consider the following indirect data-aided phase estimate:

$$
\begin{aligned}
\hat{\theta}_{k} & =\arg \left\{\widehat{r_{k}^{\prime}}\right\}=\arg \left\{\sum_{i=1}^{\nu} p_{i} r_{k-i}^{\prime}\right\} \\
& =\arg \left\{\sum_{i=1}^{\nu} p_{i} \frac{r_{k-i}}{c_{k-i}}\right\} .
\end{aligned}
$$

The optimal prediction coefficients depend in general on the state $S_{k} \triangleq\left(a_{k-1}, \ldots, a_{k-\nu}, s_{k-\nu}\right)$, whereas the MMSE depends on the corresponding transition $T_{k} \triangleq\left(a_{k}, S_{k}\right)$. Defining a trellis diagram in terms of the extended state $S_{k}$, a VA can be derived with branch metric

$$
\begin{aligned}
\gamma_{k}\left(T_{k}\right) & =\left|c_{k}\right|^{2}\left|r_{k}^{\prime}-e^{j \widehat{\theta}_{k}}\right|^{2} \\
& =\left|c_{k}\right|^{2}\left|r_{k}^{\prime}-\frac{\sum_{i=1}^{\nu} p_{i} r_{k-i}^{\prime}}{\left|\sum_{i=1}^{\nu} p_{i} r_{k-i}^{\prime}\right|}\right|^{2} .
\end{aligned}
$$

In the case of equal energy signaling, since the system matrix $\mathbf{R}$ in (6) no longer depends on $\left\{c_{k-l}\right\}_{l=1}^{\nu}$, the prediction coefficients and the MMSE do not depend on $T_{k}$, but only on the SNR.

With respect to classical linear predictive receivers for fading channels [2-5], the proposed solution features a few differences. First, the denominator in (8) normalizes the phasor estimate to unit modulus. This normalization is essential for non-equal energy signaling such as quadrature amplitude modulations (QAM). Second, the correlation matrix $\mathbf{R}$ models the statistics of the phase process, whereas in classical receivers it models the amplitude process as well.

The complexity of the proposed receivers can be limited by applying reduced-state detection techniques (see [8] and references therein). As an example, a reduced state can be defined by substituting $\nu$ with a parameter $Q<\nu$ in the definition of $S_{k}$, i.e., by memory truncation.

We remark that the prediction coefficients $\left\{p_{i}\right\}_{i=1}^{\nu}$ can also be computed by an adaptive algorithm which recursively minimizes the mean square error in (5), such as a stochastic gradient algorithm. This suggests that the proposed algorithms could be easily made adaptive by applying standard methods [9].

\section{NUMERICAL RESULTS}

A realistic model of phase noise is based on a discrete-time Wiener process $\left\{\psi_{k}\right\}$ characterized by i.i.d. Gaussian increments with zero mean and standard deviation $\sigma_{\Delta}$, descriptive of the phase noise intensity. The case of a constant random frequency offset can also be incorporated by letting $\theta_{k}=\psi_{k}+2 \pi f k T$, where $f$ is a random variable uniformly distributed in $(-\alpha / T, \alpha / T), \alpha$ is the normalized frequency offset intensity, and $T$ denotes the signaling interval. For this phase model $R_{\theta}(l)=e^{-|l| \sigma_{\Delta}^{2}} \operatorname{sinc}(2 \alpha l)$, where $\operatorname{sinc}(x) \triangleq \sin (\pi x) / \pi x$. In the absence of phase noise $\left(\sigma_{\Delta}=0\right)$ and frequency offset $(\alpha=0)$, the metric in (8) reduces to one of the noncoherent sequence detection (NSD) solutions proposed in [6] for equal energy signaling-the phase memory parameter $N$ in [6] equals $\nu+1$. Other phase models may be considered, possibly incorporating a time varying frequency offset, such as that caused by a Doppler rate [1]. For conciseness, we present results for a constant random frequency offset with phase noise only.

Fig. 2 shows the prediction coefficients as a function of the phase noise standard deviation $\sigma_{\Delta}$ for an equal energy modulation, either phase shift keying (PSK) or CPM, a prediction order $\nu=4$, and $E_{b} / N_{0}=4 \mathrm{~dB}$, where $E_{b}$ denotes the received energy per information bit. Three values of frequency offset intensity $\alpha$ are considered, namely $0,0.01$, and 0.02 . In the absence of phase instabilities $\left(\sigma_{\Delta}=0\right.$ and $\alpha=0$ ) all the prediction coefficients are equal, as expected from the equivalence with NSD. For increasing phase noise or frequency offset, the prediction coefficients take on different values-the stronger the phase variations, the larger the difference.

Differentially encoded quaternary PSK (DQPSK) is considered in Fig. 3. The performance of the communication system is assessed by computer simulations in terms of bit- 


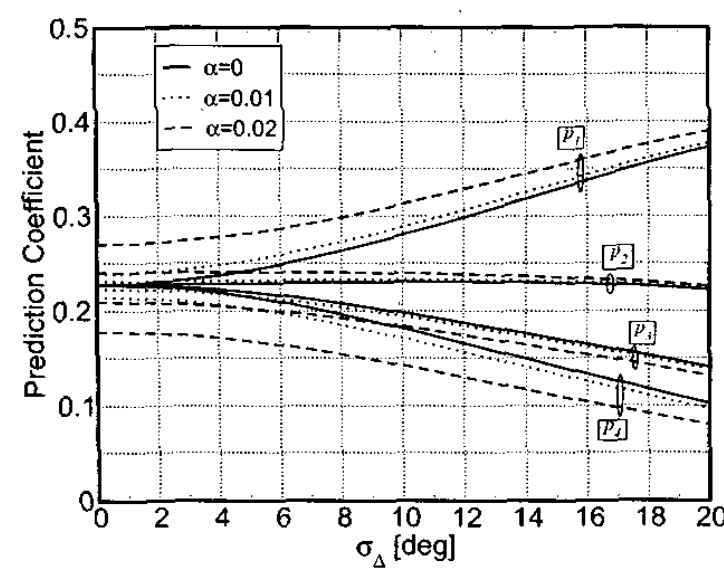

Figure 2: Prediction coefficients as a function of the phase noise standard deviation $\sigma_{\Delta}$, for an equal energy modulation, prediction order $\nu=4$, and $E_{b} / N_{0}=4 \mathrm{~dB}$. Various values of the frequency offset intensity $\alpha$ are considered.

error rate (BER) as a function of the phase noise standard deviation $\sigma_{\Delta}$ for various values of the frequency offset intensity $\alpha$ and $E_{b} / N_{0}=10 \mathrm{~dB}$. We consider symbol by symbol detection with decision feedback (i.e., $Q=0$ ). The performance of the proposed linear predictive receiver for $\nu=5$ is compared with that of the NSD algorithm in [6]. For $\alpha=0$, the curve corresponding to the proposed receiver is the "envelope" of the performance curves of the NSD algorithm for $\nu=1,2, \ldots, 5$. For any given $\sigma_{\Delta}$ and $\alpha$, there exists an optimum value of $\nu$-within the considered range-such that the BER obtained with the NSD algorithm is minimized-this optimal $\nu$ reduces for increasing values of $\sigma_{\Delta}$ and $\alpha$. The proposed linear predictive receiver with $\nu=5$ automatically minimizes the BER, provided the prediction coefficients are adaptively updated. In the presence of frequency offset, the advantage of the proposed receiver over NSD is even more pronounced, as it appears from the curves relative to $\alpha=0.02$ and $\alpha=0.05$. In the latter case, the performance of NSD schemes with $\nu>1$ is appreciably worse.

We now describe an application of the proposed detection technique to soft-output FB algorithms and iterative processing. It is possible to show, but it is beyond the scope of the paper, that the extension of the proposed linear predictive technique to FB algorithms can be devised in a systematic manner. We consider a serially concatenated convolutional code (SCCC) consisting of an outer 4-state, rate$1 / 2$ code connected through a length- 1024 pseudo-random bit-interleaver to an inner 4 -state, rate- $2 / 3$ code [10]. The output symbols are mapped to an 8-PSK constellation with natural mapping. Pilot symbols are introduced with a rate of 1 pilot every 16 information symbols. At the receiver side, the inner decoder uses the proposed linear predictive

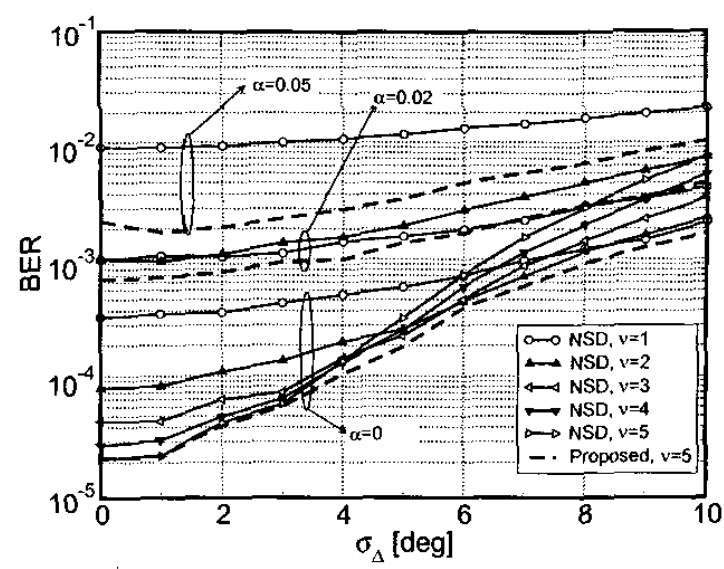

Figure 3: BER as a function of the phase noise standard deviation $\sigma_{\Delta}$ for DQPSK, symbol by symbol decision, and various values of the frequency offset intensity $\alpha$.

algorithm and performs joint detection and decoding. The numerical results are shown in Fig. 4 in terms of BER versus phase noise standard deviation, for increasing values of the frequency offset. The SNR is fixed to $4 \mathrm{~dB}$ in all cases. For $\nu=6$ and $Q=3$, the proposed iterative detection scheme is very robust to phase instabilities up to $\sigma_{\Delta}=10$ degrees and $\alpha=0.01$. A less complex receiver with $\nu=4$ and $Q=2$, has still acceptable performance for low values of $\sigma_{\Delta}$ and $\alpha$. The performance of the proposed scheme degrades significantly for $\alpha \geq 0.02$.

A CPM modulator can be decomposed into the cascade of a finite state machine (FSM) and a memoryless mapper [11]. Hence, it is immediate to extend the proposed trellis-based linear predictive receivers to this case as well. As an example, we consider a serially concatenated scheme obtained by an outer convolutional encoder and an inner Gaussian minimum shift keying (GMSK) modulator [12]. In particular, we refer to the GSM standard [13], where the outer code is a 16-state non-recursive non-systematic convolutional code with rate $1 / 2$ and generator matrix $G_{0}(D)=$ $\left[1+D^{3}+D^{4} 1+D+D^{3}+D^{4}\right]$. The outer code and the GMSK modulator are connected through a length-1024 pseudo-random bit-interleaver. The spectral efficiency of the overall code is $0.5 \mathrm{bit} / \mathrm{s} / \mathrm{Hz}$. At the receiver side we consider $\beta=2$ samples per symbol interval. The numerical results are shown in Fig. 5. As one can see, for sufficiently large $N$ and $Q$, the performance loss, with respect to an ideal coherent receiver which perfectly knows the channel phase, is within $1.2 \mathrm{~dB}$ for $\sigma_{\Delta} \leq 10$ degrees.

\section{CONCLUSIONS}

Linear predictive receivers for phase-uncertain channels have been derived. Both hard-output and soft-output detection 


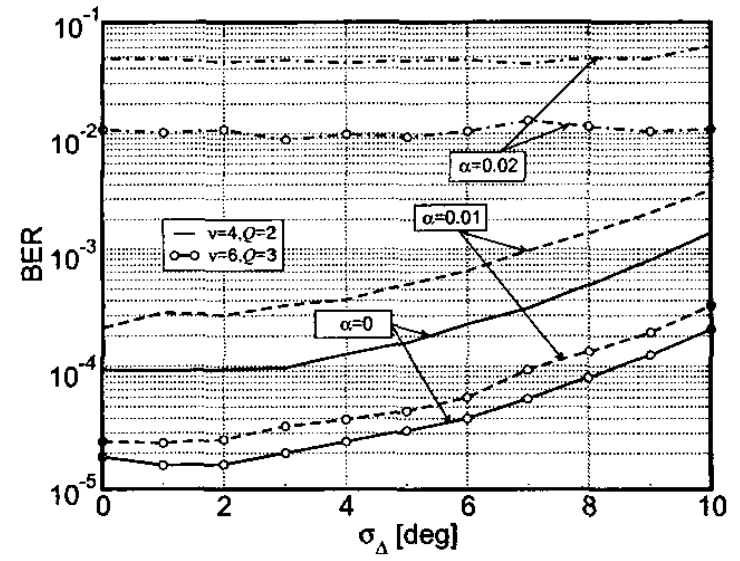

Figure 4: BER as a function of the phase noise standard deviation $\sigma_{\Delta}$ for a SCCC with 8-PSK, an inner linear predictive detector, $E_{b} / N_{0}=4 \mathrm{~dB}$, various values of frequency offset intensity and levels of receiver complexity. In all cases, 5 decoding iterations are considered.

have been considered. The proposed receivers are very robust to phase noise and uncompensated frequency offset. Adaptive versions of the proposed algorithms are attractive. As a side result, suboptimal linear predictive detection appears to be effectively applicable even in special cases with non-Gaussian observables.

\section{REFERENCES}

[1] F. Giannetti, M. Luise, and R. Reggianini, "Simple carrier frequency rate-of-change estimators," IEEE Trans. Communun., vol. 47, pp. 1310-1314, September 1999.

[2] J. Lodge and M. Moher, "Maximum likelihood estimation of CPM signals transmitted over Rayleigh flat fading channels," IEEE Trans. Communun., vol. 38, pp. 787-794, June 1990.

[3] D. Makrakis, P. T. Mathiopoulos, and D. Bouras, “Optimal decoding of coded PSK and QAM signals in correlated fast fading channels and AWGN: A combined envelope, multiple differential and coherent detection approach," IEEE Trans. Communun., vol. 42, pp. 6375, January 1994.

[4] X. Yu and S. Pasupathy, "Innovations-based MLSE for Rayleigh fading channels," IEEE Trans. Communun., vol. 43, pp. 1534-1544, February-April 1995.

[5] G. M. Vitetta and D. P. Taylor, "Maximum likelihood decoding of uncoded and coded PSK signal sequences

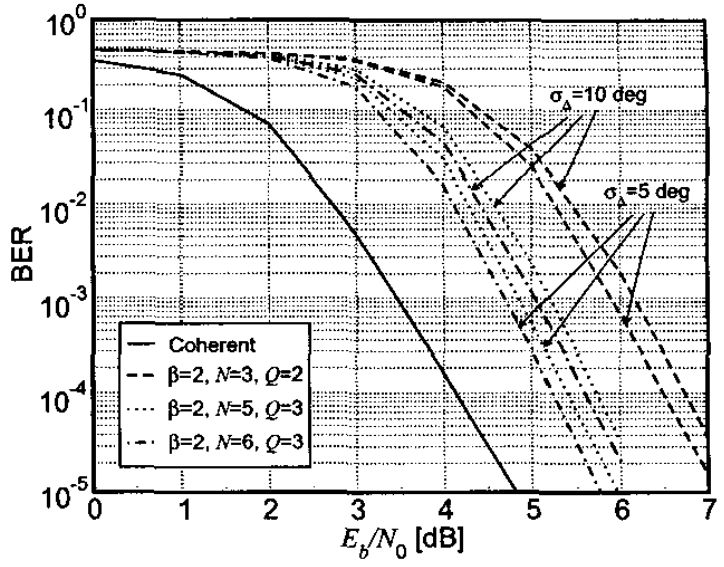

Figure 5: BER of a serially concatenated scheme with GMSK and inner linear prediction with a sampling rate of $\beta=2$ samples per symbol. Various receiver complexity levels are considered. For comparison, the performance of the coherent system is also shown. In all cases 5 decoding iterations are considered.

transmitted over Rayleigh flat-fading channels," IEEE Trans. Communun., vol. 43, no. 11, pp. 2750-2758, November 1995.

[6] G. Colavolpe and R. Raheli, "Noncoherent sequence detection," IEEE Trans. Communun., vol. 47, pp. 1376-1385, September 1999.

[7] M. Morelli and U. Mengali, "Feedforward frequency estimation for PSK: a tutorial review," European Trans. Telecommun., vol. 9, pp. 103-116, March/April 1998.

[8] G. Colavolpe, G. Ferrari, and R. Raheli, "Reducedstate BCJR-type algorithms," IEEE J. Select. Areas Commun., vol. 19, no. 5, pp. 848-859, May 2001.

[9] S. Haykin, Adaptive Filter Theory, Prentice-Hall, Englewood Cliffs, NJ, 2nd edition, 1991.

[10] G. Ferrari, A. Anastasopoulos, G. Colavolpe, and R. Raheli, "Adaptive iterative detection: a performance comparison of closed-loop and open-loop phase synchronization," in Proc. IEEE Global Telecommun. Conf., Taipei, Taiwan, November 2002.

[11] B. E. Rimoldi, "A decomposition approach to CPM," IEEE Trans. Inform. Theory, vol. 34, pp. 260-270, March 1988.

[12] J. G. Proakis, Digital Communications, McGraw-Hill, New York, 4th edition, 2001.

[13] ETSI, “ETSI EN300 909 v.8.3," July 2000. 